\title{
Automatic estimation of gestational age in ultrasound images based on direct least- squares fitting of ellipse
}

\begin{abstract}
Measurement of the fetal head biparietal diameter (BPD) and head circumference (HC) is crucial for estimation of fetal age. Due to the noisy nature of ultrasound images and variation in image acquisition and measurement techniques, manual measurements of these parameters are subject to inter- and intraobserver variability. In this study, we present an approach for estimation of gestational age in ultrasound images, based on direct least-squares fitting of an ellipse. The process goes through three steps: image preprocessing, object extraction, and fitting an ellipse to the resultant shape and measuring its parameters. The proposed technique was tested and evaluated on 20 ultrasound images of fetal head. The images had a combination of noise and low contrast. Excellent linear correlation between manual and automatic measurement was obtained, which verifies the reliability of the proposed automatic approach.
\end{abstract}

Keyword: Estimation; Gestational age; Least squares fitting; Ultrasound images 